\title{
Lidil
}

Revue de linguistique et de didactique des langues

$57 \mid 2018$

Démarches créatives, détours artistiques et appropriation des langues

\section{Marie-Cécile Guernier et Jean-Pierre Sautot (dir.), Former des adultes à l'écrit. Questions didactiques pour la professionnalisation des formateurs}

Dijon, Éditions Raisons et Passions, 2017, 186 p.

\section{Vasiliki Markaki Lothe}

\section{OpenEdition}

Journals

Édition électronique

URL : http://journals.openedition.org/lidil/4966

DOI : $10.4000 /$ lidil.4966

ISSN : 1960-6052

\section{Éditeur}

UGA Éditions/Université Grenoble Alpes

Édition imprimée

ISBN : 978-2-37747-048-8

ISSN : 1146-6480

Référence électronique

Vasiliki Markaki Lothe, « Marie-Cécile Guernier et Jean-Pierre Sautot (dir.), Former des adultes à l'écrit. Questions didactiques pour la professionnalisation des formateurs », Lidil [En ligne], 57 | 2018, mis en ligne le 01 mai 2018, consulté le 24 septembre 2020. URL : http://journals.openedition.org/lidil/4966 ; DOI : https://doi.org/10.4000/lidil.4966

Ce document a été généré automatiquement le 24 septembre 2020.

(C) Lidil 


\title{
Marie-Cécile Guernier et Jean-Pierre Sautot (dir.), Former des adultes à l'écrit. Questions didactiques pour la professionnalisation des formateurs
}

\author{
Dijon, Éditions Raisons et Passions, 2017, 186 p.
}

Vasiliki Markaki Lothe

\section{RÉFÉRENCE}

Marie-Cécile Guernier et Jean-Pierre Sautot (dir.), Former des adultes à l'écrit. Questions didactiques pour la professionnalisation des formateurs, Dijon, Éditions Raisons et Passions, $2017,186 \mathrm{p}$.

Quels sont les enjeux et les conditions de réussite pour le développement d'une collaboration entre des praticiens formateurs d'adultes et des chercheurs s'intéressant à la formation? En quoi et comment les ajustements entre les deux parties influent sur les manières à la fois de représenter et d'explorer une problématique commune autour de la formation des adultes à l'écrit? Quelles sont les compétences requises pour la conduite de ce type de formations? Au moyen d'une écriture synthétique et accessible, l'ouvrage dirigé par Marie-Cécile Guernier et Jean-Pierre Sautot retrace les étapes de construction d'un projet de recherche-action-formation sur les formateurs d'adultes avant de s'atteler à l'exposition d'une collection de travaux issus de ce projet. La présentation du projet offre ainsi en premier lieu un précieux témoignage sur les contraintes qui pèsent sur une démarche de recherche participative (chapitres 1 et 2). Elle offre aussi des résultats de recherche qui ne se dérobent pas devant la question de la complexité du contexte d'un tel projet et par conséquent des défis méthodologiques auxquels les chercheurs et leurs collaborateurs scientifiques ont été confrontés (chapitres 3 à 6). 
Initié dans le cadre de la lutte contre l'illettrisme, le groupe de travail GRAFFIC (Groupe Recherche Action Formation Français Insertion Compétences) réunit des chercheurs et des acteurs de la formation s'intéressant à l'appropriation de l'écrit par les adultes. Lieu de questions et d'organisation d'actions collectives sur les compétences clés des formateurs d'adultes, le groupe GRAFFIC propose plusieurs méthodes d'enquête (entretiens, questionnaires, grilles d'observation) et des concepts-outils d'évaluation et de modélisation de l'activité menée pour l'apprentissage de l'écrit. Cette richesse méthodologique et conceptuelle est le point de départ de l'adaptation de nombreux outils d'analyse, autant de clés de compréhension, destinés aux formateurs et présentés dans l'ouvrage. Pour orienter la production de ces outils, inspirés des questionnements en didactique et pouvant contribuer à la professionnalisation des formateurs, les membres du groupe GRAFFIC multiplient les observations et les entretiens avec les formateurs et les apprenants. En plus des outils et des méthodes mobilisées, les auteurs développent aussi me semble-t-il avec ce livre un style d'écriture particulier pour présenter des résultats, mêlant avec habileté les références théoriques aux références de terrain, le tout dans des textes allant droit au but. Ce faisant, ils tentent de répondre aux attentes d'un lectorat diversifié propre à un travail interdisciplinaire/inter-métiers et nous invitent à réfléchir de manière plus générale à la nécessité de penser l'écriture scientifique dans le cadre d'un projet collaboratif, en déterminant ses spécificités et sa portée.

3 Les recherches du groupe mettent en lumière aussi bien la diversité des profils des formateurs et des apprenants que la complexité de l'activité du formateur et des processus d'apprentissage en jeu chez l'adulte. Elles documentent ainsi l'activité des formateurs dans le domaine de la formation des adultes à l'écrit et contribuent à la reconnaissance des spécificités de leur métier et donc à leur professionnalisation. Par ailleurs, les chapitres de cet ouvrage mettent en lumière les corrélations existantes entre les spécificités du métier des formateurs, les dimensions socio-historique et sociocognitive des situations de formation et la transmission des savoirs. Ces distinctions expliquent que derrière les difficultés que rencontrent les formateurs en situation de travail, dans le décalage qui existe entre ce qui est prescrit et ce qui est réalisé, se confrontent des conceptions variées de l'activité de la formation et de ses objectifs. En documentant ces conceptions, l'ouvrage réussit à élargir l'éventail des études empiriques faites sur les formateurs d'adultes et à apporter une contribution scientifique nouvelle sur la question de la formation professionnelle des formateurs.

\section{AUTEURS}

\section{VASILIKI MARKAKI LOTHE}

Laboratoire LARAC, Université Grenoble Alpes 